\title{
O Aprendizado através de um Jogo Colaborativo-Competitivo contra Dengue
}

\author{
Diego Buchinger, Marcelo da Silva Hounsell
}

Programa de Pós Graduação Stricto Sensu em Computação Aplicada (PPGCA) Universidade do Estado de Santa Catarina (UDESC), Joinville - Santa Catarina - Brasil

diego.buchingerdoutlook.com, marcelo.hounsellaudesc.br

\begin{abstract}
The dengue fever disease is a public health problem in many countries and, currently, the praxis for controlling this disease is raising the population awareness about this problem. Thus, the use of Serious Games with collaborative and competitive interactions altogether could increase motivation and provide learning. A group of 43 children/teenagers played the game Sherlock Dengue 8 and their learning was evaluated. The results have shown increase of $19 \%$ on knowledge and $42 \%$ on confidence about the subject, and also, a moderate relationship between the in-game score and learning has found. The collected data indicate that a collaborativecompetitive game has the potential to be an effective tool for dengue learning.
\end{abstract}

Resumo. A dengue vem se mostrando um problema de saúde pública em muitos países e a prática mais adotada para o controle desta doença é a conscientização da população. Assim, propõe-se o uso de Jogos Sérios com interações colaborativas e competitivas juntas como um meio de aumentar a motivação e trazer aprendizado. Avaliou-se a aprendizagem associada ao uso do jogo Sherlock Dengue 8 com um grupo de 43 crianças/adolescentes, obtendo-se um aumento médio de 19\% no conhecimento e $42 \%$ na confiança sobre o assunto, além de uma correlação moderada entre a pontuação obtida no jogo e o aprendizado. Os dados obtidos indicam que um jogo colaborativocompetitivo tem potencial de ser eficaz para o aprendizado sobre dengue.

\section{Introdução}

Uma das doenças que vem preocupando a população mundial, principalmente nos países tropicais e subtropicais é a dengue. A doença da dengue é uma das arboviroses que mais mata no mundo, sendo que o número de casos notificados da doença continua a crescer, inclusive no Brasil [Guzman e Istúriz, 2010]. A dengue é uma doença transmitida por mosquitos do gênero Aedes: o Aedes aegypti e o Aedes albopictus, sendo que o vírus se instala no mosquito quando este pica um ser infectado pela doença [Rajapakse et al., 2012].

Atualmente, pesquisas estão sendo realizadas a fim de criar uma vacina para a doença a fim de imunizar as pessoas [Ratnam et al., 2013]. Enquanto uma vacina efetiva não for produzida, a medida mais eficaz é o combate à reprodução do mosquito transmissor, através da eliminação ou tratamento dos possíveis locais que possam servir como depósito de ovos dos mosquitos vetores da doença. Entretanto, para combater o mosquito da dengue desta forma, é necessária a colaboração de toda a população, e por isso é necessário investir em campanhas de conscientização. 
A conscientização de crianças e adolescentes, contudo, vem se mostrando como uma tarefa complexa. Os ditos Nativos Digitais - ou geração $Z$ - veem o ensino tradicional como entediante e pouco motivador, pois estão acostumados - e possuem gosto - por aplicações multimídia e novidades tecnológicas [Prensky, 2003]. Dessa forma, pesquisadores sugerem o uso de Jogos Sérios (JS - Serious Games) como jogos que são criados especificamente para serem utilizados como ferramentas de ensino, adicionando conteúdos educacionais no design do jogo, mas não abrindo mão do aspecto de entretenimento [Susi et al., 2007].

Estudos recentes têm indicado que a integração de ambientes colaborativos e competitivos pode ser eficaz para aprendizagem [Fong-Ling et al., 2009]. Assim, criouse um novo Jogo Sério Colaborativo-Competitivo (JSCC) que abordasse a temática da doença da dengue e, com base neste jogo, avaliou-se a sua eficácia na aprendizagem sobre a doença. Este artigo mostrará a fundamentação do jogo, a forma de avaliação adotada, os resultados obtidos e suas análises.

\section{Trabalhos Relacionados}

A busca por trabalhos relacionados foi realizada inicialmente através da procura de JSCC sobre dengue, contudo nenhum trabalho foi encontrado. Assim, subdividiu-se a pesquisa em duas ramificações: uma procurando por JSCC abordando qualquer temática e outra procurando por jogos sobre dengue.

Através de uma revisão de literatura sobre JSCC foram encontrados 17 artigos que apresentavam aspectos de 12 jogos diferentes (i.e. alguns artigos tratavam de um mesmo jogo). O mapeamento desta pesquisa foi apresentado por Buchinger e Hounsell (2013), onde se destacou que não foi encontrada nenhuma guideline para a elaboração de JSCC. Entretanto, as características de cada jogo apresentado, junto das experiências ressaltadas nos 17 artigos, serviram como base para a discussão posterior do game design do JSCC sobre dengue que viria a ser desenvolvido.

$\mathrm{Na}$ segunda etapa da pesquisa verificou-se que, excluindo as pesquisas em medicina, não existem muitos trabalhos científicos e JS voltados para a temática da dengue. Dentre os trabalhos encontrados na pesquisa, destaca-se a iniciativa da academia, na elaboração de JS sobre dengue, principalmente nos países tropicais e subtropicais - que são mais atingidos pela doença - e também de empresas, através do desenvolvimento de jogos web voltados principalmente para o entretenimento.

Nas Filipinas, Lennon e Coombs (2007) realizaram um estudo sobre a influência de um jogo de tabuleiro ("Goodbye-to-Dengue" - GD) no processo de aprendizagem da dengue. $\mathrm{O}$ jogo utiliza-se de cartas interativas que apresentam informações sobre dengue e o conhecimento adquirido é reforçado no jogo através de perguntas. Durante este estudo, foi realizada uma análise de eficácia de aprendizagem, atitudes-crenças e auto eficácia. Dividiram-se os participantes em dois subgrupos: um que utilizou apenas o jogo como fonte de conhecimento e outro que teve apenas aulas teóricas sobre o assunto. $\mathrm{O}$ resultado indicou que o grupo que teve apenas aulas obteve melhores resultados de aprendizagem, enquanto que atitudes-crenças e auto eficácia aumentaram em ambos os grupos de maneira não significativa. Lennon e Coombs (2007) argumentam que o grupo que teve apenas aulas sofreu grande influência das habilidades de ensino do professor, e que no caso específico do experimento realizado, os professores escolhidos eram os melhores avaliados nas escolas. 
Na Venezuela, Vivas e Guevara de Sequeda (2003) realizaram um estudo sobre a eficácia de um jogo ("Jugando em Salud: dengue" - JSD) como ferramenta de aprendizado, controle e prevenção contra a dengue no ensino às crianças que estudam no ensino fundamental. A eficácia do jogo foi testada através de um experimento envolvendo três grupos: um grupo que usou o jogo três vezes por semana durante 60 dias e seus professores receberam novos materiais teóricos sobre a doença; outro grupo que recebeu apenas os novos materiais; e o último grupo que seguiu apenas o programa de ensino regular (grupo de controle). Todos foram avaliados através de questionários antes e após o período do programa, sendo verificado um aumento significativo no conhecimento sobre dengue nos dois primeiros grupos, e na aceitação do jogo.

No Brasil, Silva et al. (2011) apresentaram a proposta de um Role Playing Game (RPG) sobre dengue que enfatiza também a cultura e a geografia do nordeste brasileiro. O jogo ("Exterminadores de Dengue" - ED) põe o jogador sobre o controle de um personagem que tem um de seus parentes infectados pelo vírus da doença. $\mathrm{O}$ jogador possui os objetivos de eliminar os mosquitos Aedes Aegypti em batalhas no estilo RPG, eliminar os depósitos do mosquito, além de procurar e trazer medicamentos para o parente infectado. Também no Brasil, Pereira et al. (2011) apresentaram o jogo "Contra Dengue" (CD). Neste jogo, os jogadores devem explorar o ambiente, passando por obstáculos, eliminando os possíveis depósitos para o mosquito vetor, e procurando por itens que restauram seus pontos de vida e que forneçam pontos. Ambos os jogos são voltados para crianças e adolescentes, e nenhum estudo sobre seu uso foi mencionado.

Outro projeto que vem sendo desenvolvido no Brasil é o Sherlock Dengue (SD) [Sherlock Dengue, 2015]. Este projeto envolve o desenvolvimento de JS utilizando diferentes tecnologias e design, mas mantendo o foco na divulgação e ensino de informações sobre a dengue de maneira interativa. $O$ projeto conta com oito versões distintas (sendo a oitava versão tópico deste artigo), voltadas principalmente para crianças e adolescentes, com exceção da sétima versão que tem como público-alvo os especialistas da área da saúde.

Para sumarizar os dados dos estudos ressaltados é apresentada a Tabela 1. Note que foram incluídas apenas as versões dois e seis do projeto Sherlock Dengue, porque somente nestas duas versões foram realizados estudos avaliativos. Pode-se observar a predominância de crianças e adolescentes como público alvo, o uso de questionários para coleta de dados e uma tendência na avaliação de aprendizagem e aceitação dos jogos. Pode-se considerar que a avaliação de aceitação é uma forma de verificar a motivação dos participantes, pois a aceitação é um pré-requisito da motivação.

Existem também vários jogos não apresentados na literatura científica (e.g. Contra a Dengue 2, Colorir, Dengue, Dengue Buster, Hugo Contra Dengue) geralmente com estilo e aparência de aplicativos Flash 2D - que abordam o tema da doença da dengue, mas possuem e apresentam pouco conteúdo sobre a doença, dando maior ênfase para os aspectos interativos do jogo. Em alguns casos, inclusive, não há um vínculo direto entre as informações sobre a dengue e a jogabilidade: a informação é apresentada separadamente do jogo.

Nenhum dos jogos encontrados adota um design colaborativo-competitivo que, entende-se ser uma alternativa engajadora para a aprendizagem. Então, apesar de não ser tão frequente a avaliação da aprendizagem, este trabalho analisa este aspecto para um jogo de natureza colaborativa-competitiva. Entretanto, para diminuir o impacto das 
respostas pelo método de "chute", incluiu-se a possibilidade dos participantes relatarem sua confiança na resposta, sendo que este dado também foi avaliado.

Tabela 1. Características dos trabalhos relacionados

\begin{tabular}{|c|c|c|c|c|c|c|}
\hline Características & GD* & JSD* & ED* & CD* & $S D 2 *$ & $S D 6^{*}$ \\
\hline Público Alvo & $\begin{array}{c}\text { Crianças e } \\
\text { adolescentes }\end{array}$ & Crianças & $\begin{array}{l}\text { Crianças e } \\
\text { adolescen- } \\
\text { tes }\end{array}$ & $\begin{array}{c}\text { Crianças e } \\
\text { adolescentes }\end{array}$ & $\begin{array}{c}\text { Crianças e } \\
\text { adolescentes }\end{array}$ & $\begin{array}{c}\text { Crianças, } \\
\text { adolescentes } \\
\text { e especialistas } \\
\text { em saúde }\end{array}$ \\
\hline $\begin{array}{l}\text { Número de } \\
\text { Participantes }\end{array}$ & $\begin{array}{l}168 \text { crianças e } \\
\text { adolescentes }\end{array}$ & $\begin{array}{l}621 \text { alunos } \\
\text { entre } 8 \text { e } 16 \\
\text { anos }\end{array}$ & - & - & $\begin{array}{l}71 \text { alunos } \\
\text { entre } 9 \text { e } 24 \\
\text { anos }\end{array}$ & $\begin{array}{l}32 \text { alunos } \\
\text { entre } 18 \text { a } 39 \\
\text { anos }\end{array}$ \\
\hline $\begin{array}{c}\text { Métodos para } \\
\text { Coleta de } \\
\text { Dados }\end{array}$ & Questionário & Questionário & - & - & Questionário & Questionário \\
\hline $\begin{array}{l}\text { Realizou pré e } \\
\text { pós-teste? }\end{array}$ & Sim & Sim & - & - & Não & Não \\
\hline $\begin{array}{l}\text { O que foi } \\
\text { Avaliado? }\end{array}$ & Aprendizagem & $\begin{array}{l}\text { Aprendizagem } \\
\text { e aceitação do } \\
\text { jogo }\end{array}$ & - & - & $\begin{array}{c}\text { Aceitação do } \\
\text { jogo }\end{array}$ & $\begin{array}{c}\text { Aceitação do } \\
\text { jogo e } \\
\text { colaboração }\end{array}$ \\
\hline
\end{tabular}

* GD - Goodbye-to-Dengue JSD - Jugando em Salud: dengue; ED - Exterminadores de Dengue; CD - Contra Dengue; SD - Sherlock Dengue;

\section{Metodologia Científica}

Para as etapas inicias de pesquisa foram realizadas pesquisas bibliográficas com o intuito de conhecer os trabalhos relacionados e aprofundar o conhecimento nas etapas associadas ao desenvolvimento de um JSCC sobre dengue. Em uma segunda etapa foram realizadas pesquisas exploratórias que fomentaram discussões sobre o game design do jogo e em sequência a etapa de desenvolvimento do jogo. Posteriormente foram realizados testes pilotos - para verificar o funcionamento do jogo - realização de alguns ajustes e, por fim, fez-se uso do jogo em uma única seção de até duas horas. Para mensurar o aprendizado foi desenvolvido um instrumento avaliativo aplicado antes (préteste) e após (pós-teste) o uso do jogo. Os resultados obtidos foram compilados e analisados com uso de estatística descritiva e indutiva.

\subsection{Materiais: O Jogo Sério Sherlock Dengue 8 The Neighborhood}

Tomando-se como base o game design legado da série Sherlock Dengue [Sherlock Dengue, 2015], e o material revisado durante a busca por trabalhos correlatos, elaborouse o game design de um novo JSCC sobre dengue: o Sherlock Dengue 8 The Neighborhood. O público alvo selecionado é composto por crianças e adolescentes entre 9 e 16 anos. O jogo foi desenvolvido para computador, contando com versões para Windows, Linux (inclusive Linux Educacional utilizado nas escolas do Brasil) e MacOS, sendo que a versão mais recente do jogo permite tanto partidas colaborativocompetitivo (2 versus 2), assim como partidas somente colaborativas, somente competitivas, ou ainda, individuais.

O Sherlock Dengue 8 é um jogo 3D online do tipo investigativo e arcade, no qual os jogadores assumem o papel de inspetores da dengue e podem participam de inspeções (partidas). No modo colaborativo-competitivo, chamado no jogo de duelo de pares, duas duplas de jogadores se enfrentam em uma partida. Os jogadores devem 
encontrar e eliminar os depósitos utilizados pelo mosquito transmissor da dengue que estão espalhados pelo cenário virtual a fim de ganhar pontos. Todavia, para eliminar tais depósitos, deve-se responder a uma pergunta sobre a doença. A fím de obter o conhecimento necessário para responder as perguntas, os jogadores devem encontrar livros e tablets que contém informações sobre a doença da dengue. Através da leitura dessas informações, os inspetores terão o conhecimento para responder as perguntas quando encontrarem os depósitos, e assim ganhar pontos.

Ressalta-se que o jogo desenvolvido é gratuito e possui materiais de apoio ao jogador (manual) e ao professor (plano de ensino). Para utilizá-lo, deve-se realizar apenas um cadastro, através do próprio jogo. Outras funcionalidades adicionais presentes no jogo são: (a) modo tutorial, que ensina a como jogar no próprio jogo; (b) conquistas, os jogadores ganham medalhas por realizarem determinadas ações no jogo; (c) ranking para cada modo de jogo e subdividido em categorias de escolaridade; e (d) disponibilidade de dez avatares. Maiores detalhes sobre o projeto e o funcionamento do jogo podem ser verificados em Sherlock Dengue (2015).

\subsection{Métodos}

Com o propósito de realizar uma avaliação de aprendizagem foi elaborado um questionário de conhecimento sobre dengue, baseado em algumas das informações que são apresentadas no decorrer do jogo. $O$ questionário consiste em dez questões de múltipla escolha, de níveis de dificuldade variado, sendo que cada questão possui quatro alternativas de resposta. Além disso, a fim de evitar possíveis resultados inconsistentes devido ao método de "chute", teve-se a preocupação de avaliar o grau de confiança nas respostas dos participantes. Dessa forma, cada questão possui um espaço reservado para que o participante indique qual o grau de confiança na resposta registrada. $O$ questionário pode ser visualizado no manual do jogo [Sherlock Dengue, 2015].

Para realizar a avaliação e obter os dados foram promovidos mutirões de conscientização sobre dengue no município de Joinville (Santa Catarina) com o públicoalvo, contando com o apoio da Secretaria Municipal de Saúde da cidade. Os mutirões incluíam as seguintes etapas principais:

(1) Explicações de quais atividades seriam realizadas durante o mutirão;

(2) Aplicação do instrumento avaliativo (pré-teste)

(3) Utilização do jogo: (a) cadastro dos participantes no jogo, (b) uso do modo treino, que ensina o funcionamento do jogo (gameplay) e seus mecanismos, por 10 a 20 minutos, e (c) uma partida colaborativa-competitiva (2vs2) do jogo com duração de 60 minutos;

(4) Nova aplicação do instrumento avaliativo (pós-teste).

Deve-se salientar que, em momento algum é mencionado aos participantes que o mesmo instrumento avaliativo seria utilizado novamente após o uso do jogo, fato que poderia comprometer os resultados obtidos.

Ao total, 43 alunos entre o $7^{\circ}$ ano do Ensino Fundamental (EF) ao $3^{\circ}$ ano do Ensino Médio (EM) participaram dos mutirões realizados. A idade média destes alunos era de 13,56 anos, com desvio padrão de 1,28 anos, sendo que $18,60 \%$ estudavam no $7^{\circ}$ ano do EF; $11,63 \%$ estudavam no $8^{\circ}$ ano do EF; $46,51 \%$ estudavam no $9^{\circ}$ ano do EF; $16,28 \%$ estudavam no $1^{\circ}$ ano do EM; e $6,98 \%$ estudavam no $3^{\circ}$ ano do EM. 


\section{Resultados e Análise}

O desempenho de cada indivíduo no questionário avaliativo foi mensurado em uma escala de zero a dez, sendo que cada pergunta do questionário tinha o peso de um ponto. O gráfico da Figura 1 sintetiza os resultados obtidos pelos participantes no pré-teste e no pós-teste, indicando a ocorrência das notas finais de cada participante.

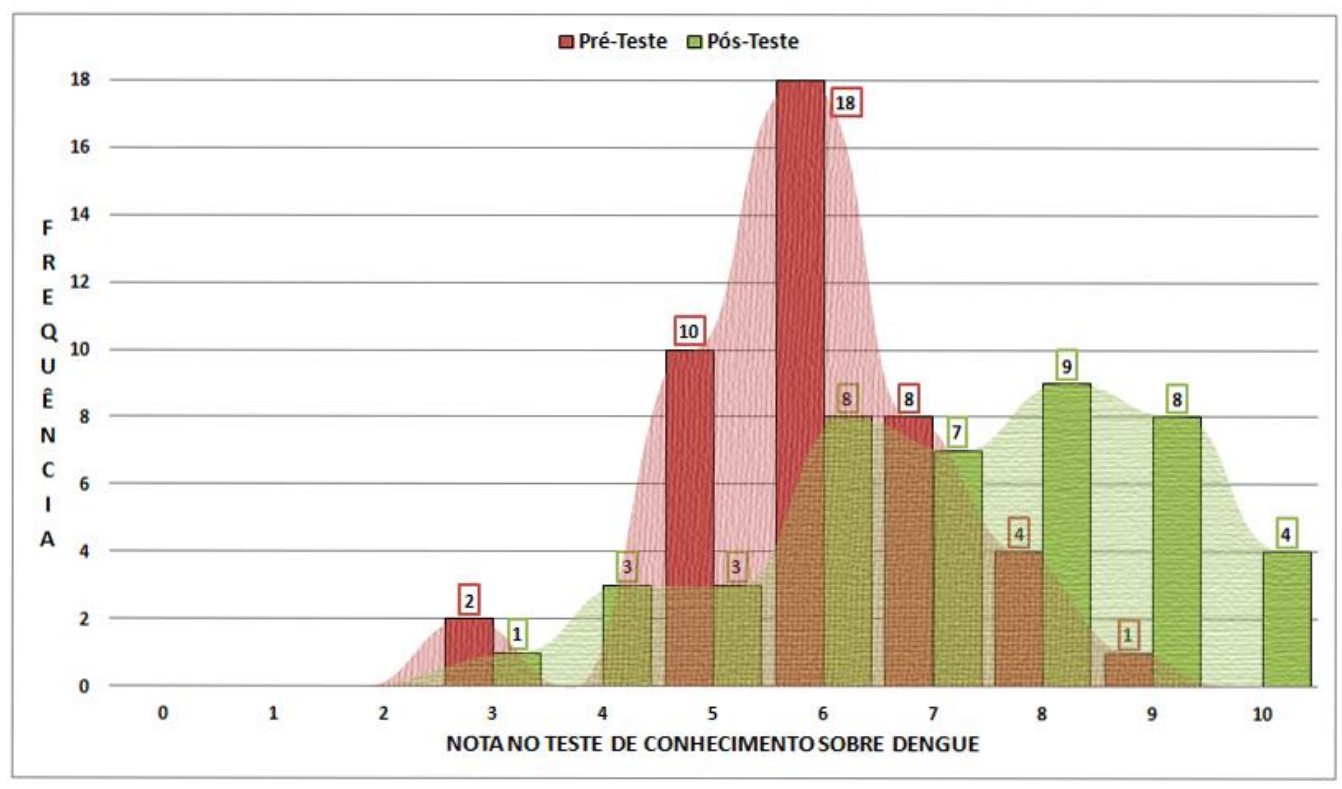

Figura 1. Quantidade de acertos no questionário de conhecimento

Pode-se perceber através do gráfico da Figura 1 que os participantes obtiveram melhores notas no pós-teste do que no pré-teste. Apurou-se que $60,70 \%$ das questões foram respondidas corretamente pelos participantes no pré-teste, ao passo que $72,33 \%$ das questões foram respondidas corretamente no pós-teste. Além disso, 30,23\% dos participantes obtiveram nota igual ou superior a sete no pré-teste - nota considerada como limiar em aprovação em muitas escolas - enquanto que $65,12 \%$ dos participantes conseguiram o mesmo no pós-teste. Vale salientar ainda que nenhum participante obteve a nota máxima no pré-teste, mas $9,3 \%$ conseguiram obter tal nota no pós-teste.

Com base nos dados coletados durante a avaliação de desempenho, puderam ser sintetizados os seguintes dados: o desempenho médio $(\overline{\mathrm{x}})$ durante o pré-teste foi de 6,07 pontos, com desvio padrão amostral de $\mathrm{s}=1,20$, mediana $\mathrm{Md}=6$ e moda $\mathrm{Mo}=6$, ao passo que o desempenho médio $(\overline{\mathrm{x}})$ durante o pós-teste foi de 7,23 pontos, com $\mathrm{s}=1,81$, $\mathrm{Md}=7$ e $\mathrm{Mo}=8$. Utilizando-se da estatística indutiva, calculou-se ainda a estimativa para uma média populacional da nota de pós-teste, considerando os dados coletados. Foi utilizado um nível de confiança de $99 \%$ para as 43 amostras, obtendo-se o seguinte intervalo: $6,49 \leq \mu \leq 7,98$. Além disso, calculou-se por fim uma estimativa para a diferença entre as médias populacionais das notas de pré-teste e pós-teste. Foi utilizado novamente um nível de confiança de $99 \%$ para os dois grupos de 43 amostras, obtendose o seguinte intervalo de diferença: $0,29 \leq \mu_{\text {pos }}-\mu_{\text {pre }} \leq 2,04$, i.e., se novos testes fossem realizados em condições semelhantes, em $99 \%$ das vezes a média das notas do pós-teste seria maior em 0,29 a 2,04 pontos do que a média das notas do pré-teste.

Decidiu-se também analisar o comportamento do desempenho na avaliação de acordo com a escolaridade dos participantes. Assim, elaborou-se a Figura 2 que 
apresenta a relação entre escolaridade dos participantes e o desempenho no questionário de conhecimento aplicado antes e após a utilização do Sherlock Dengue 8. As linhas sólidas da Figura 2 indicam as médias dos grupos por escolaridade, ao passo que, as linhas tracejadas e as áreas com hachura, indicam o desvio padrão destas médias.

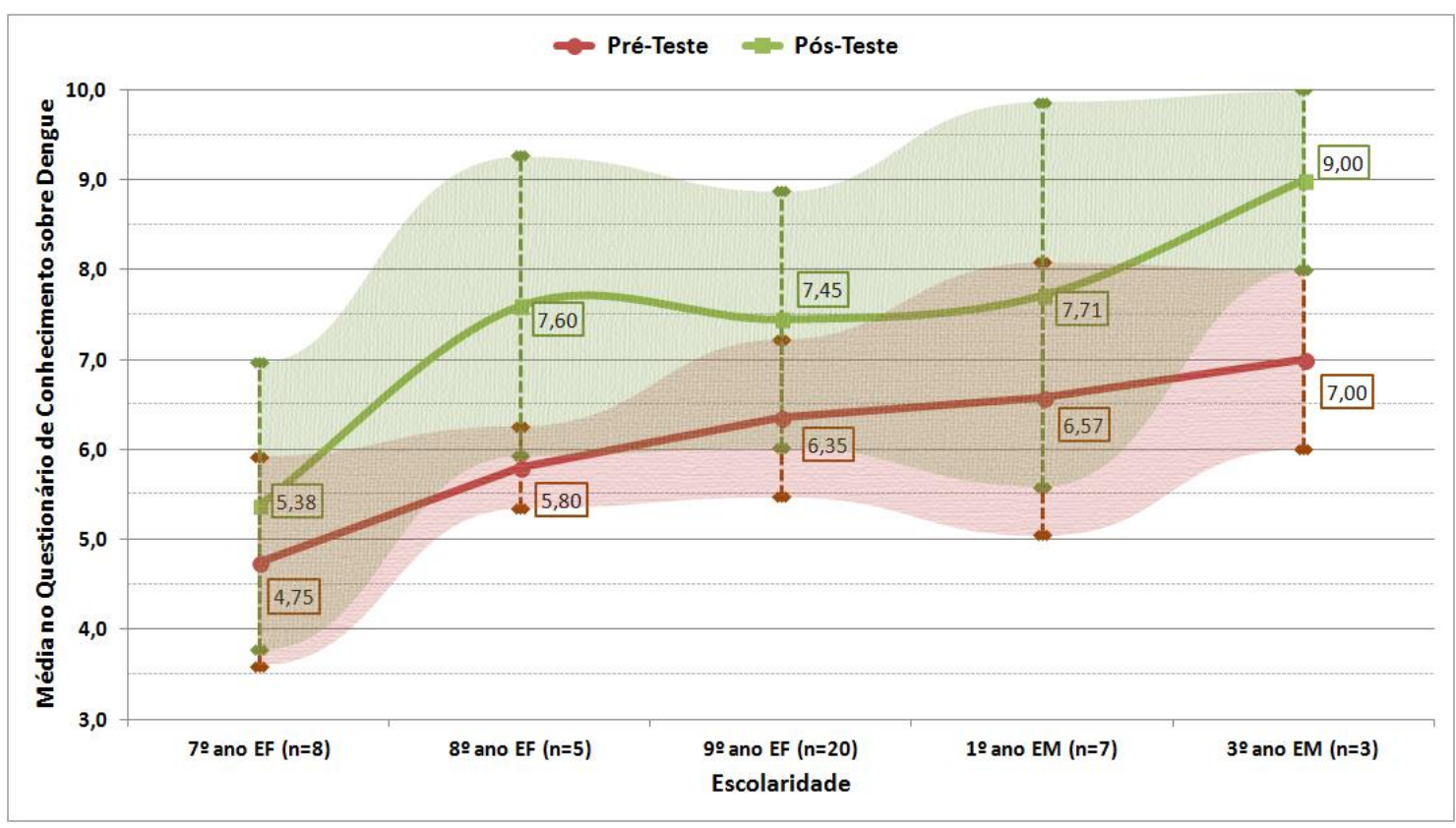

Figura 2. Desempenho no questionário de conhecimento por escolaridade

Pode-se perceber através da Figura 2 que, para todas as escolaridades, houve um aumento da média das notas obtidas no questionário de conhecimento. Deve-se ressaltar ainda o comportamento crescente das médias obtidas durante o pré-teste, indicando a diferença de conhecimento esperado entre alunos de escolaridades maiores. O mesmo comportamento ocorreu na média de pós-teste, com exceção da média do $9^{\circ}$ ano. Devese levar em consideração, contudo, o número de amostras de cada escolaridade, que estão diretamente relacionados à confiabilidade da generalização ou inferência através dos resultados.

\subsection{Relação entre Confiança nas Respostas e o Jogo}

$\mathrm{O}$ aspecto da confiança nas respostas dadas ao questionário de conhecimento também foi avaliado quantitativamente com base em uma escala de zero a dez. Inicialmente foram calculadas as médias e desvios padrão para cada questão do pré-teste e do pósteste (Figura 3), levando em consideração a confiança nas respostas corretas e incorretas. Pode-se perceber que o nível de confiança médio para cada questão sempre esteve maior no pós-teste do que no pré-teste. Depois, calculou-se a confiança média geral, obtendo-se: $\overline{\mathrm{x}}=6,00(\mathrm{~s}=3,21)$ para o pré-teste e $\overline{\mathrm{x}}=8,54(\mathrm{~s}=2,25)$ para o pósteste, indicando um aumento médio de confiança na ordem de $42,35 \%$.

Analisando os dados de maneira mais aprofundada, foram verificadas as seguintes informações:

- $50,47 \%$ dos participantes responderam corretamente uma mesma pergunta tanto no pré-teste quanto no pós-teste. Destes: $32,72 \%$ aumentaram sua confiança na resposta; 59,45\% mantiveram uma mesma confiança na resposta; e 7,83\% diminuíram sua confiança na resposta; 
- $21,86 \%$ dos participantes erraram uma resposta no pré-teste, mas responderam corretamente no pós-teste. Destes: $8,51 \%$ aumentaram sua confiança na resposta; $86,17 \%$ mantiveram uma mesma confiança na resposta; $5,32 \%$ diminuíram sua confiança na resposta;

- $10,23 \%$ dos participantes responderam corretamente uma pergunta no pré-teste, mas erraram a resposta no pós-teste. Destes: $29,54 \%$ aumentaram sua confiança na resposta; 54,55\% mantiveram uma mesma confiança na resposta; $15,91 \%$ diminuíram sua confiança na resposta.

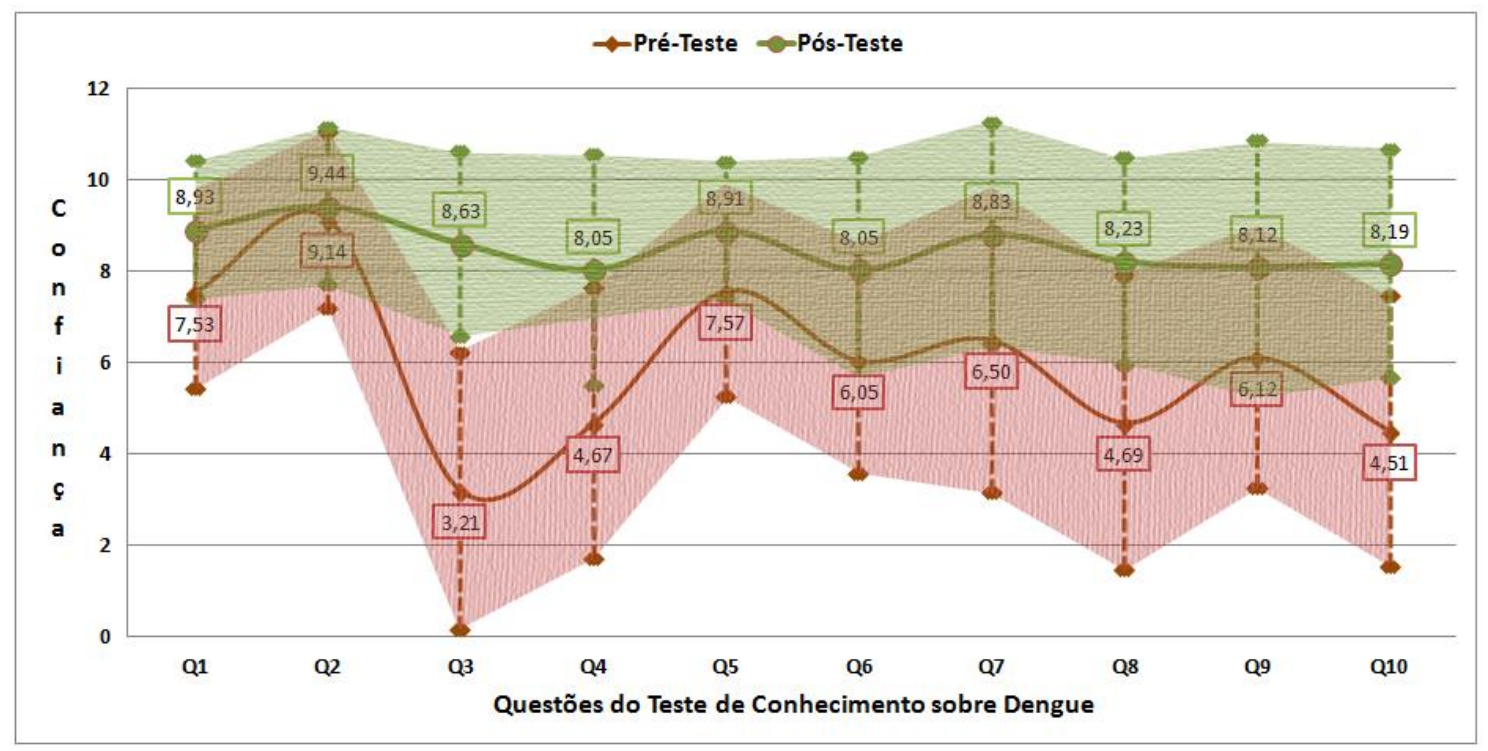

Figura 3. Relação de confiança na resposta no pré-teste e pós-teste

Os dados da confiança nas respostas ressaltam que, na maioria dos casos, a confiança dos participantes se manteve a mesma em diferentes situações. Em muitas vezes porém, a confiança dos participantes em uma dada resposta correta aumentou, indicando que o jogo pode ter ajudado na confiança sobre o tópico abordado. Por outro lado, percebeu-se que houve aumento de confiança mesmo em respostas erradas, o que pode indicar um mau entendimento da informação apresentada, ressaltando a necessidade de atividades de reforço com um professor em sala de aula.

\subsection{Relação entre Notas e o Jogo}

Avaliou-se também a relação entre a nota no pós-teste e o desempenho no jogo (pontos conquistados no jogo durante uma partida colaborativa-competitiva). Para isso, elaborou-se o gráfico da Figura 4 que, além de apresentar os dados, também indica a que grupo de escolaridade cada registro pertence. A partir do gráfico é possível observar que existe uma correlação entre as variáveis, de tal modo que, em média, os jogadores que conquistaram maiores pontuações no jogo, obtiveram maiores notas no pós-teste.

A fim de obter valores quantitativos de correlação, foram calculados os valores de correlação linear $(r=0,4162 ; y=0,0056 x+4,8814)$, correlação logarítmica $(r=0,4695 ; y=2,50171 \times \ln (x))$ e correlação exponencial $(r=0,5135$; $\left.y=0,5366 \times x^{0,4291}\right)$. Os valores calculados para o modelo linear e logarítmico indicam uma correlação fraca $(|r|<0,5)$ entre a pontuação em jogo e a nota obtida no pós-teste, ao passo que, o valor da correlação exponencial indica uma correlação moderada $(0,5 \leq|r|<0,7)$ entre estas variáveis. Como o modelo exponencial explica 
melhor a correlação, adicionou-se uma linha tracejada no gráfico da Figura 4 que indica a linha dessa tendência.

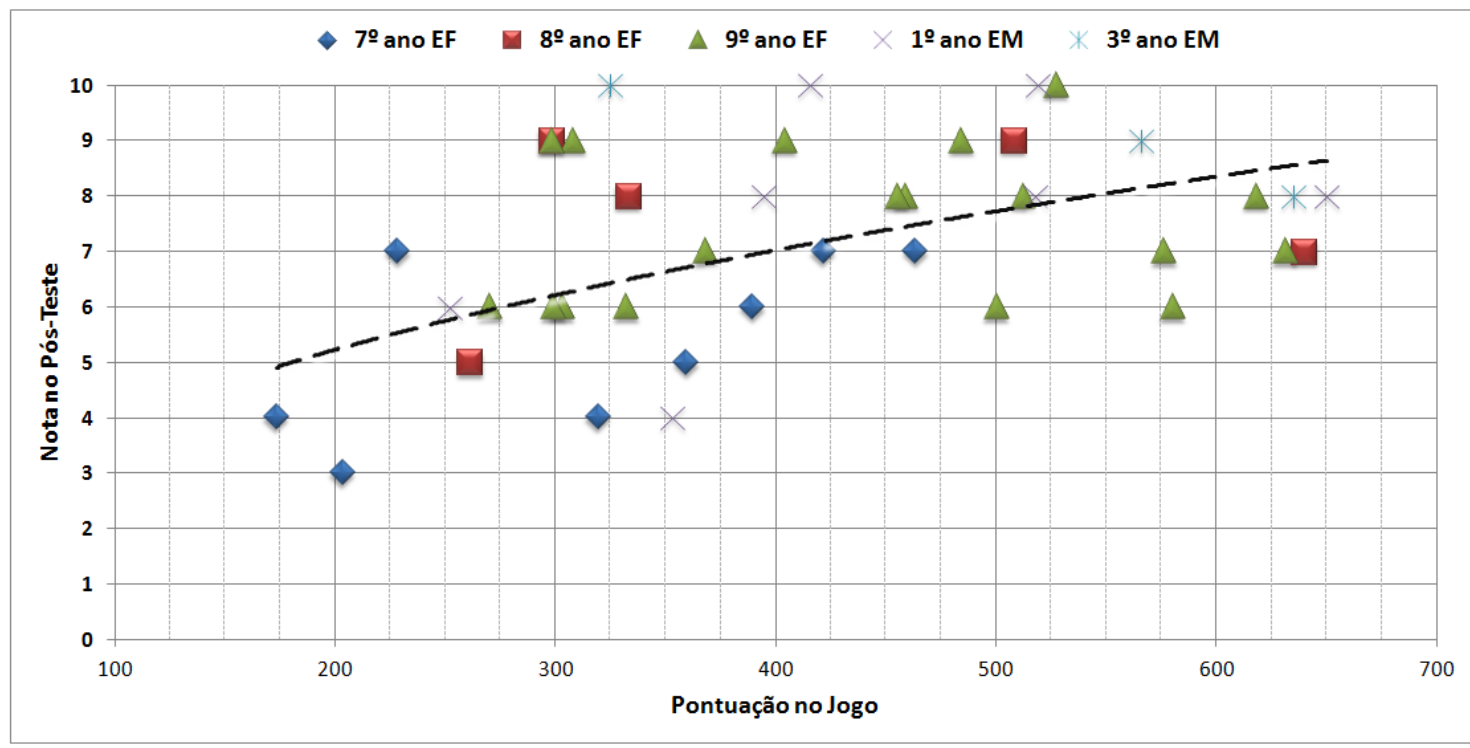

Figura 4. Relação entre pontuação obtida no jogo e nota no pós-teste

Como o modelo exponencial indicou uma correção moderada entre a pontuação obtida em jogo e a nota obtida no pós-teste, argumenta-se que seria possível utilizar o próprio jogo como ferramenta de aprendizagem e avaliação de aprendizagem sobre dengue. Uma vez que os jogadores que conquistarem maiores pontuações durante o jogo, supostamente acertariam mais respostas em uma avaliação sobre dengue com o mesmo conteúdo, seria possível substituir a nota de uma prova escrita por uma nota relacionada à pontuação obtida durante uma partida do jogo.

\section{Conclusões}

A mortalidade relacionada à dengue continua a crescer. Enquanto que uma vacina de imunização não for desenvolvida, devem-se adotar medidas de prevenção e combate ao mosquito transmissor da doença. Para conscientizar as crianças e adolescentes da nova geração, uma alternativa que tem apresentado resultados positivos é a utilização de jogos, e pesquisas recentes têm indicado que o modo de interação colaborativo e competitivo, juntos, em atividades diversas, pode promover maior aprendizado.

A fim de verificar se um Jogo Sério Colaborativo-Competitivo (JSCC) sobre dengue pode realmente ser eficaz na aprendizagem deste assunto, foi desenvolvido o Sherlock Dengue 8 The Neighborhood. A eficácia do jogo no aprendizado sobre a dengue para crianças e adolescentes foi avaliada através de um questionário, que foi utilizado para verificar o nível de conhecimento e confiança nas respostas, antes e após o uso do jogo. Obteve-se aumento de $19,16 \%$ nas notas das avaliações de conhecimento e $42,33 \%$ na confiança de que as respostas assinaladas estavam corretas.

Com base nos resultados obtidos com uma amostra de 43 pessoas, é possível sugerir que o JSCC construído realmente auxilia no processo de aprendizado sobre dengue, aumentando o nível de conhecimento e a confiança no assunto. Inclusive, encontrou-se uma correlação exponencial moderada $(r=0,5135)$ entre a pontuação obtida em jogo e a nota obtida no questionário de conhecimento, após o uso do jogo. 
Deve-se ressaltar contudo, a importância da presença do professor e de atividades complementares para reforçar e confirmar o conhecimento adquirido com o uso do jogo.

\section{Agradecimentos}

Os autores gostariam de agradecer à Universidade do Estado de Santa Catarina pela bolsa de estudos (PROMOP) concedida, à FITEJ e à Secretaria de Saúde de Joinville.

\section{Referências}

Buchinger, D., Hounsell, M. S. (2013) Jogos Sérios Competitivo-Colaborativos: Um Mapeamento Sistemático da Literatura. In: XXIV Simpósio Brasileiro de Informática na Educação - SBIE, p. 275-284.

Buchinger, D., Hounsell, M. S. (2014) Sherlock Dengue 8: A Serious Game for Teaching about Dengue Fever Prevention with Collaboration and Competition. In: XIII Simpósio Brasileiro de Jogos e Entretenimento Digital - SBGAMES, Trilha de Cultura, p. 400-409.

Fong-Ling, F., Ya-Ling, W., His-Chuan, H. (2009) An Investigation of Coopetitive Pedagogic Design for Knowledge Creation in Web-Based Learning. In: Computers \& Education, 53(3):550-562.

Guzman, A., Istúriz, R. E. (2010) Update on the Global Spread of Dengue. In: International Journal of Antimicrobial Agents, 36(1):S40-S42.

Lennon, J. L., Coombs, D. W. (2007) The Utility of a Board Game for Dengue Haemorrhagic Fever Health Education. In: Health Education, 107(3):290-306.

Pereira, P. F., Silva, R. B., Bareato, R., Camargo, T. C., Bittar, T. J., Longo, E. (2011) Considerações para jogos de ação do tipo plataforma com base nas experiências do desenvolvimento do jogo Contra Dengue. In: X Simpósio Brasileiro de Jogos e Entretenimento Digital - SBGAMES, Trilha de Computação, 4 p.

Prensky, M. (2003) Digital Game-Based Learning. In: ACM Computers in Entertainment, 1(1), 4 p.

Rajapakse, S., Rodrigo, C., Rajapakse, A. (2012) Treatment of Dengue Fever. In: Journal of Infection and Drug Resistance, 5:103-112.

Ratnam, I., Leder, K., Black, J., Torresi, J. (2013) Dengue Fever and International Travel. In: Journal of Travel Medicine, 20(5), 10 p.

Sherlock Dengue. (2015) Disponível em: http://www2.joinville.udesc.br/ larva/dengue/

Silva, V., Almeida, A., Alves, D. da S.; Andrade, M., Araujo, A. (2011) Exterminadores de Dengue: Um jogo educativo dinâmico como ferramenta de educação contra a dengue. In: X Simpósio Brasileiro de Jogos e Entretenimento Digital - SBGAMES, Trilha de Cultura, 4 p.

Susi, T., Johannesson, M., Backlund, P. (2007) Serious Games - An Overview. In: Technical Report HS- IKI -TR-07-001, 28 p.

Vivas, E., Guevara de Sequeda, M. (2003) A Game as an Educational Strategy for the Control of Aedes Aegypti in Venezuelan school children. In: Pan American Journal of Public Health, 14(6):394-401. 